\title{
Levantamento do nível de conhecimento dos manipuladores de serviços de alimentação em feiras livres sobre Boas Práticas de Fabricação, na Cidade de Vitória da Conquista - BA
}

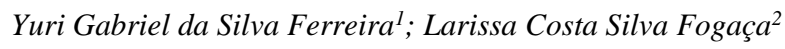

\begin{abstract}
Resumo: As feiras livres são locais de socialização e fácil acesso, se configurando pela variedade de alimentos e produtos oferecidos além de preços mais acessíveis. Nestes locais de alimentação encontram-se os manipuladores de alimentos, que desempenham papel importante em reduzir os riscos de contaminação alimentar evitando assim as Doenças Transmitidas por Alimentos - DTA. Com o presente estudo objetivou-se avaliar o nível de conhecimento dos manipuladores de alimentos em feiras livres na cidade de Vitória da Conquista - BA, sobre as boas práticas de fabricação, verificando se estes seguem os padrões estipulados pela RDC no 216 de 2004 . Realizou-se uma pesquisa de amostragem não probabilística intencional, no período de fevereiro a março de 2018 , onde as pessoas entrevistadas foram exclusivamente os manipuladores de alimentos, e os resultados foram avaliados de acordo com parâmetro de classificação deficiente, regular e bom. Dos 20 manipuladores entrevistados $45 \%$ não tem conhecimento sobre boas práticas, sendo classificados como deficientes, e 55\% possuem, sendo classificados como regulares. Em relação à capacitação apenas $25 \%$ dos manipuladores receberam algum tipo de curso e os outros $75 \%$ não. Os resultados indicam a necessidade de um maior investimento na capacitação destes manipuladores, com uma maior frequência de cursos, utilizando uma didática que leve esse conhecimento de forma simplificada para maior entendimento dos mesmos, e que atenda a legislação sanitária vigente nestes estabelecimentos.
\end{abstract}

Palavras-chave: Manipuladores de alimentos, boas práticas de fabricação, capacitação.

\section{Survey of the level of knowledge of food service handlers in open fairs on Good Manufacturing Practices, in the City of Vitória da Conquista, Bahia}

\begin{abstract}
The free fairs are places of socialization and easy access, being configured by the variety of food and products offered in addition to more affordable prices. At these feeding sites are the food handlers, who play an important role in reducing the risks of food contamination thus avoiding Foodborne Diseases - DTA. This study aimed to evaluate the level of knowledge of food handlers in open markets in the city of Vitória da Conquista BA, on good manufacturing practices, verifying if they follow the standards stipulated by RDC $n^{\circ} 216$ of 2004 . An intentional non-probabilistic sampling was carried out from February to March 2018, where the persons interviewed were exclusively food handlers, and the results were evaluated according to poor, regular and good classification parameters. Of the 20 handlers interviewed, $45 \%$ do not know about good practices, being classified as disabled, and $55 \%$ have, being classified as regular. Regarding training only $25 \%$ of the handlers received some type of course and the other $75 \%$ did not. The results indicate the need for a greater investment in the training of these manipulators, with a higher frequency of courses, using a didactics that simplifies this knowledge for a better understanding of them, and that meets the sanitary legislation in force in these establishments.
\end{abstract}

Keywords: Food handlers, good manufacturing practices, training.

${ }_{1}^{1}$ Graduando do curso de Farmácia pela Faculdade Independente do Nordeste - FAINOR. yuri.gabriel03@ gmail.com;
${ }^{2}$ MSc. em Engenharia de Alimentos - Professora do Colegiado de Farmácia - FAINOR

1093 Id on Line Rev. Mult. Psic. V.12, N. 40. 2018 - ISSN 1981-1179

Edição eletrônica em http://idonline.emnuvens.com.br/id 


\section{Introdução}

$\mathrm{Na}$ atualidade as feiras livres são locais de socialização e comercialização de produtos de qualidade com preço acessível. No decorrer das constantes mudanças na vida cotidiana das pessoas, a alimentação fora de casa se torna uma opção mais rápida e prática para saciar a fome (GOLIN et al.,2017). Alguns fatores que podem influenciar na busca por esse alimento são a distância entre casa e trabalho associada à falta de transporte particular e pouco tempo para horário de almoço. Estes são alguns pontos que fazem com que as pessoas recorram às feiras livres por não terem um local fixo para refeição (SANTINI; SEIXAS, 2016).

A infraestrutura inadequada, qualidade dos produtos oferecidos, poucas frequências de fiscalização contribuem diretamente para qualidade dos alimentos ofertados nas ruas. Estas condições agregadas ao nível de conhecimento do manipulador, que é o principal veículo de transporte dos microrganismos, contribuem ao não cumprimento das boas práticas de fabricação, aumentando os riscos das Doenças Transmitidas por Alimentos - DTA (SOUZA et al.,2015). As DTA são patologias de origem microbiana, que se dão através de água e alimentos contaminados, geralmente proveniente da falta de capacitação e condições higiênico-sanitárias dos manipuladores e estabelecimentos respectivamente (BRASIL, 2017).

Para Agostinho (2013), as DTA indicam um problema de saúde pública, porém a sua quantificação de surtos é dificultada pela não obrigatoriedade de notificação, ocasionando um deficiente perfil epidemiológico no Brasil, ao contrário dos EUA, em que, anualmente são registrados cerca de 76 milhões de casos, destes, 325 mil são enfermos e 5 mil vão a óbito. Como no Brasil não é obrigatória à notificação, alguns estados e municípios armazenam dados sobre os agentes causadores mais comuns, alimento mais frequente, e fatores de risco. Cerca de 2 milhões de indivíduos vão a óbito por ano, devido a distúrbios diarreicos, que na maioria dos casos tem como seu vetor inicial, os alimentos contaminados. Vários são os fatores que influenciam nos surtos das DTAs podendo destacar as condições ao exportar alimentos, e o aumento da população.

A formação dos manipuladores de alimentos deve ser imprescindível, pois esta é uma estratégia que visa à garantia de segurança dos alimentos (SOARES et al., 2016). A regulamentação dos manipuladores e do estabelecimento se dá através da RDC na 216 de 2004, em que os mesmos devem estar em condições favoráveis para garantia do produto acabado. No 
decorrer de toda resolução é enfatizada a importância sobre a lavagem das mãos sempre que for manusear alimento. Essa lavagem deve ser realizada de maneira correta, estipulada pela lei e o estabelecimento deve conter cartazes autoexplicativos. As mãos são o principal vetor de contaminação e propagação de microrganismos, e o manipulador deve ter total conhecimento sobre as doenças causadas por estes, porém isso só é observado quando é realizada uma capacitação adequada que deve ser comprovada mediante documentação (BRASIL, 2004).

Com o presente trabalho objetivou-se avaliar o nível de conhecimento dos manipuladores de alimentos em feiras livres na cidade de Vitória da Conquista - BA sobre as boas práticas de fabricação verificando se estes seguem os padrões estipulados pela RDC $\mathrm{n}^{\circ}$ 216 de 2004

\section{Metodologia}

O estudo realizado para o levantamento do conhecimento dos manipuladores de serviços de alimentação em feiras livres trata-se de uma pesquisa do tipo exploratória, descritiva, analítica qualitativa e quantitativa dos dados obtidos, tendo como campos base para coleta de dados os vários estabelecimentos que servem comida pronta localizados em Mercados Municipais, situados nos bairros Centro e Brasil na cidade de Vitória da Conquista, Bahia.

Foi realizada uma pesquisa de amostragem não probabilística intencional, onde as pessoas entrevistadas foram exclusivamente os manipuladores de alimentos. Como critério de exclusão foram descartados os vendedores ambulantes por não terem ponto fixo, não se enquadrando nos requisitos da RDC no 216 de 2004.

A análise dos dados coletados foi realizada com critérios estabelecidos para inclusão e exclusão da pesquisa, onde os dados foram trabalhados em forma percentual de acordo as respostas obtidas.

A análise das boas práticas de manipulação se deu através do preenchimento de questionário adaptado de MELLO et al (2010), direcionado aos manipuladores com informações pertinentes a pesquisa, tais como: idade, escolaridade, capacitação, conhecimento sobre contaminação de alimentos, doenças causadas por alimentos, boas práticas de manipulação e higiene das mãos. 
O parâmetro para classificação do nível de conhecimento sobre contaminação dos alimentos bem como das doenças transmitidas pelos mesmos e, das boas práticas de manipulação, foi adaptado de Saccol (2007), que se dividiu em 3 grupos: grupo 1, grupo 2 e grupo 3 sendo respectivamente, deficiente: de 0 a 50\%; regular: de 51 a 75\%; e bom de 76 a $100 \%$ das respostas corretas.

A pesquisa dispôs de computadores com suporte de senha para proteção dos dados entrevistados, bem como, a garantia do sigilo, onde não foram utilizados aparelhos celulares para armazenamento de informações referentes aos entrevistados. A pesquisa obteve aprovação do comitê de ética da Faculdade Independente do Nordeste, com protocolo de aprovação n ${ }^{\circ}$ 2.418.864 para a coleta de dados da pesquisa, preservando assim, a privacidade e a confidencialidade dos participantes analisados pela pesquisa.

\section{Resultados e Discussão}

Foram entrevistados 20 manipuladores de alimentos, um por estabelecimento, que trabalham em ponto fixo, situados em feiras livres, nos bairros Centro e Brasil, na cidade de Vitória da Conquista, Bahia, no período de fevereiro a março de 2018. A tabela 1 apresenta a distribuição da amostra de acordo a faixa etária.

Tabela1: Distribuição etária dos manipuladores de alimentos em serviços de alimentação em feiras livres, na cidade de Vitória da Conquista - BA.

\begin{tabular}{c|cc}
\multirow{2}{*}{ FAIXA ETÁRIA } & \multicolumn{2}{c}{ FREQUÊNCIA } \\
\cline { 2 - 3 } & $\mathrm{N}$ & $\%$ \\
\hline ATÉ 20 ANOS & 0 & 0 \\
20- 29 ANOS & 6 & 30 \\
30-39 ANOS & 5 & 25 \\
40-49 ANOS & 5 & 25 \\
ACIMA DE 50 ANOS & 4 & 20 \\
TOTAL & 20 & - \\
\hline
\end{tabular}

Fonte: Dados da pesquisa 
Neste estudo, pode se observar que $30 \%$ da população entrevistada encontra-se na faixa etária entre 20 a 29 anos, assemelhando-se aos dados da pesquisa de CASTRO, BARBOSA e TABAI (2011) em que ocorreu predomínio de manipuladores entre 20 a 24 anos, aproximadamente $28,8 \%$. Observou-se que a faixa etária, tal como a experiência desses, não se configura como fator determinante para o exercício da profissão de manipulador de alimentos, assim como o grau de escolaridade, em que houve predominância do ensino fundamental incompleto (35\%), como disposto na Tabela 2.

Tabela 2: Grau de escolaridade dos manipuladores de alimentos em serviços de alimentação em feiras livres, na cidade de Vitória da Conquista - BA.

FREQUENCIA

\begin{tabular}{c|cc} 
GRAU DE ESCOLARIDADE & N & $\%$ \\
\cline { 2 - 3 } & 7 & 35 \\
\hline FUNDAMENTAL INCOMPLETO & 6 & 30 \\
FUNDAMENTAL COMPLETO & 3 & 15 \\
MÉDIO INCOMPLETO & 4 & 20 \\
MÉDIO COMPLETO & 20 & 100 \\
TOTAL & &
\end{tabular}

Fonte: Dados da pesquisa

Em relação à escolaridade, foi observado um número maior de manipuladores que não haviam concluído o ensino fundamental (35\%), e que apenas $20 \%$ concluíram o ensino médio, corroborando estes dados aos encontrados por MELLO e colaboradores. (2010), em que há a predominância de baixo nível de escolaridade em manipuladores de alimentos. Por mais que, na atualidade, o grau de ensino seja um quesito essencial na contratação de funcionários, podese perceber que há um maior número de manipuladores com ensino fundamental incompleto, comprometendo diretamente a segurança na manipulação dos alimentos, devido ao seu conhecimento reduzido (CAVALI; SALAY, 2007).

Diante da predominância do baixo grau de escolaridade dos manipuladores, observouse que o nível de conhecimento acerca das diversas fontes de contaminação de alimentos também foi deficiente (30\%), conforme disposto na Tabela 3. 
Tabela 3: Conhecimento dos manipuladores sobre as principais fontes de contaminação dos alimentos

\begin{tabular}{c|cc}
\multicolumn{1}{c}{ FONTES DE CONTAMINAÇÃO } & \multicolumn{2}{c}{ FREQUENCIA } \\
\cline { 2 - 2 } $\begin{array}{c}\text { MICRORGANISMOS (BACTÉRIAS, } \\
\text { VÍRUS E FUNGOS) }\end{array}$ & $\mathrm{n}$ & $\%$ \\
SUJEIRA & 8 & 40 \\
FALTA DE HIGIENE & 2 & 10 \\
NÃO SABEM & 4 & 20 \\
TOTAL & 6 & 30 \\
\hline
\end{tabular}

Fonte: Dados da pesquisa

Como observado na Tabela 3, o número de manipuladores que não sabem como ocorre a contaminação de alimentos é predominante, o que causa um sério risco a saúde pública. Segundo MELLO e colaboradores (2010) o não conhecimento sobre a forma de contaminação de alimentos, implica a necessidade de uma capacitação eficaz, seguida de uma assistência ao manipulador na realização de suas atividades.

Uma das formas de contaminação dos alimentos é a falta de higiene pessoal, em que os microrganismos se aproveitam dessas condições sanitárias para se instalar no alimento, causando assim a doenças transmitidas por alimentos (DTA's), sendo que os manipuladores que tem contato direto com o alimento, são mais propícios a causar essas contaminações (ANDREOTTI et al 2003).

Em um estudo realizado por SILVA e colaboradores (2005), os principais causadores de doenças transmitidas por alimentos são respectivamente vírus, bactérias e parasitas, e que a principal causa destas contaminações se dá através da manipulação indevida de alimentos. Como apresentado na tabela 3,70\% dos manipuladores sabiam como ocorriam a infecção, enquadrando seus respectivos estabelecimentos como regulares e $30 \%$ não sabiam como ocorre, sendo classificados como deficientes.

Dentre os pontos de higiene pessoal, está a lavagem das mãos, e neste trabalho foi apontado o grau de importância dos manipuladores em relação a lavagem, como disposto na Tabela 4. 
Tabela 4: Percepção do grau de importância da lavagem das mãos pelos manipuladores de alimentos.

IMPORTÂNCIA

FREQUÊNCIA

\begin{tabular}{c|cc}
\cline { 2 - 3 } & $\mathrm{n}$ & $\%$ \\
\hline CONSIDERAM IMPORTANTE & 18 & 90 \\
NÃO CONSIDERAM & 2 & 10 \\
TOTAL & & 20 \\
\end{tabular}

Fonte: Dados da pesquisa

Tabela 5: Frequência de lavagem das mãos dos manipuladores de alimentos

FREQUÊNCIA

\begin{tabular}{c|c|c}
\cline { 2 - 3 } NÃO LAVAM & N & $\%$ \\
LAVAM DE 1 A 3 VEZES & 5 & 25 \\
LAVAM DE 4 A 6 VEZES & 4 & 20 \\
LAVAM MAIS DE 7 VEZES & 8 & 40 \\
TOTAL & 2 & 10 \\
\hline
\end{tabular}

Fonte: Dados da pesquisa

De acordo a RDC 216/04, as mãos devem sempre ser lavadas antes e após o manuseio de alimentos, quando utilizar o sanitário ou ao manusear quaisquer outros equipamentos, e sempre que for necessário (BRASIL, 2004).

A higienização das mãos é de suma importância em estabelecimentos que comercializam alimentos, pois estas são veículos para o transporte de microrganismos para o alimento (ZANDONADI e colaboradores 2007). Foi observado na tabela 4 que 90\% manipuladores têm conhecimento acerca da importância da lavagem das mãos, sendo que 40\% realizam a higiene das mãos de 4 a 6 vezes ao longo do dia e $25 \%$ não lavam as mãos ao manipularem os alimentos como disposto na tabela 5. No estudo de COSTA e colaboradores (2012), foi observado que apenas $8 \%$ dos manipuladores realizam higiene das mãos antes da manipulação de alimentos e principalmente após qualquer interrupção. Segundo MAYRA e 
colaboradores 2014, as mãos são a principal causa de contaminação de alimentos devido a carga microbiológica presente, quando não há a higienização correta.

$\mathrm{Na}$ tabela a seguir, os manipuladores foram questionados sobre algumas doenças causadas pela falta de higiene das mãos.

Tabela 6. Principais doenças causadas por alimentos segundo os manipuladores.

\begin{tabular}{c|cc}
\multicolumn{1}{c}{ Doença } & \multicolumn{2}{c}{ Frequência } \\
\cline { 2 - 3 } & $\mathrm{N}$ & $\%$ \\
\hline DIARRÉIA & 4 & 20 \\
INFECÇÃO & & 20 \\
INTESTINAL & 4 & 5 \\
H. PYLORI & 1 & 5 \\
DOR DE & 2 & 10 \\
BARRIGA & & \\
VÔMITO & 1 & 5 \\
VERMES & 1 & 5 \\
SALMONELLA & 1 & 5 \\
VIROSE & 1 & 5 \\
NÃO SABEM & 5 & 25 \\
TOTAL & $\mathbf{2 0}$ & $\mathbf{1 0 0}$
\end{tabular}

Fonte: Dados da pesquisa

Apenas $10 \%$ dos manipuladores $(n=2)$ responderam corretamente sobre o proposto na tabela 6, afirmando que as doenças causadas por alimentos são oriundas das bactérias Salmonella spp e H. Pylori. O resultado indica que o conhecimento dos manipuladores a respeito das doenças transmitidas por alimentos é deficiente, pelo falo de que apenas $10 \%$ dos manipuladores tem conhecimento sobre as fontes de infecção. O restante dos manipuladores (90\%), confundiu doença com sintomas gerais, como dor de barriga, vômito e infecção intestinal, dados semelhantes aos encontrados por MELLO e colaboradores (2010).

Segundo o Ministério da Saúde (2018) as doenças transmitidas por alimentos são oriundas de água ou alimentos, de mesma procedência, contaminados por microrganismos, 
sendo eles bactérias, parasitas e vírus, provocando os mesmos sinais e sintomas em indivíduos diferentes. A RDC 216/04 determina que a contaminação de alimentos possa ocorrer pelo não uso de equipamentos de proteção individual, e que os manipuladores devem fazer uso dos mesmos ou adotar medidas que reduzam o risco de contaminação (BRASIL, 2004). Na tabela 7 estão apresentados os resultados para o uso de EPI pelos manipuladores de alimentos.

Tabela 7: Uso de Equipamento de Proteção Individual (EPI) pelos manipuladores.

USO DOS EPI'S TREQUENCIA N N $\quad$ F $\%$

\begin{tabular}{c|c}
\hline USAM & $12(60)$ \\
NÃO USAM & $8(40)$ \\
TOTAL & $20(100)$ \\
\hline
\end{tabular}

Fonte: Dados da pesquisa

Segundo a RDC 216/04 os manipuladores de alimentos devem minimizar o risco de contaminação ao alimento, e um dos meios para evitar isso é o uso dos equipamentos de proteção individual. Esse parâmetro foi analisado no ato da entrevista e foi observado que $40 \%$ dos manipuladores não utilizavam todos os EPI no momento, e dos que utilizavam, apenas foi evidenciado o uso de toucas, o que foi observado também no estudo de Oliveira e Gonçalves (2015) que constataram que $75 \%$ dos manipuladores entrevistados em sua pesquisa não utilizavam nenhum outro EPI a não ser a touca e ainda assim no ato de preparo do alimento. $\mathrm{Na}$ tabela 8 relacionou-se à capacitação dos manipuladores, com o conhecimento destes sobre as boas práticas de manipulação.

Tabela 8: Conhecimento sobre boas práticas de manipulação e capacitação dos manipuladores.

CONHECIMENTO SOBRE

BOAS PRÁTICAS

TOTAL

\begin{tabular}{c|cccccc}
\hline CAPACITAÇÃO & \multicolumn{2}{|c}{ SIM } & $\mathrm{n}$ & NÃO & $\mathrm{n}$ & $\%$ \\
CAPACITADOS & 3 & 15 & 2 & 10 & 5 & 25 \\
NÃO CAPACITADOS & 8 & 40 & 7 & 35 & 15 & 75 \\
\end{tabular}

Fonte: Dados da pesquisa 
Diante dos resultados apresentados observa-se que apenas $25 \%$ dos entrevistados alegaram terem sido capacitados e dentre estes, apenas $60 \%$ possuem conhecimento sobre as boas práticas de fabricação. A capacitação dos manipuladores é essencial para aprimorar seu conhecimento sobre a qualidade e segurança dos alimentos, higiene pessoal além da saúde própria e coletiva. Segundo a RDC 216/04 os manipuladores de alimentos devem ser sujeitos a cursos de capacitação. Estes cursos devem ser comprovados com a apresentação de documentação e devem, no mínimo, ser abordados quesitos como: boas práticas, manipulação higiênica dos alimentos, doenças transmitidas por alimentos e contaminantes alimentares, capacitando minimamente estes manipuladores em sua atuação de forma segura nos serviços de alimentação.

Dentre os entrevistados, $75 \%$ afirmaram não terem sido capacitados para o manuseio correto de alimentos, entretanto 53,3\% alegaram conhecimento sobre as boas práticas de fabricação, sugerindo assim que o conhecimento alegado não foi fundamentado por profissionais da área que estão aptos a transmitir as informações pertinentes à produção segura dos alimentos.

\section{Considerações Finais}

Diante dos resultados observa-se a importância dos cursos de capacitação aos manipuladores de alimentos, transmitindo a estes os conhecimentos necessários sobre as boas práticas de fabricação, portanto é importante que essa capacitação seja realizada com uma didática que atenda às necessidades, visto que os manipuladores que não concluíram o ensino fundamental podem apresentar dificuldades durante esta capacitação, afim de obter um bom aproveitamento, visto que o número de manipuladores que não recebeu nenhum tipo de capacitação foi de $75 \%$.

Nenhum dos questionamentos feitos foi classificado como bom, no entanto o nível de conhecimento dos manipuladores, em geral, sobre: boas práticas, higiene pessoal, importância da lavagem das mãos e uso de EPI's, fontes de contaminação e doenças transmitidas, é regular, o que pode promover riscos à garantia de qualidade do alimento, acarretando as DTA.

Recomenda-se que os órgãos de fiscalização se mobilizem sendo mais efetivos, realizando fiscalizações periódicas, promovendo incentivos e investimento na capacitação 
destes profissionais, com a finalidade de garantir a segurança dos alimentos e redução das doenças causadas por eles.

\section{Referências}

GOLIN, Anieli et al. Qualidade higiênico-sanitária e o perfil de feirantes e usuários das feiras-livres de Santa Maria - RS. Disciplinarum Scientia. Série: Ciências da Saúde, Santa Maria, v. 17, n. 3, p. 423-434, 2017.

SANTINI, V; SEIXAS, F. R. F. Avaliação das condições higiênico-sanitárias de restaurantes comerciais da cidade de Rolim de Moura - RO. Revista da UNESC, v. 14, n. 1 (2016).

BRASIL, Secretaria de Vigilância Sanitária. Doenças Transmitidas por Alimentos (DTA). Portal da Saúde - Ministério da Saúde. 2017. Disponível em: www.saude.gov.br. Data do acesso: 21 de Outubro de 2017.

AGOSTINHO, Tânia Maria de Souza. Perfil do risco sanitário de alimentos comercializados em feiras especiais de Goiânia-GO. Goiânica, 2013. Disponível em: http://bdm.unb.br/bitstream/10483/522/1/2004_DanielMarchioriSilva\%20Rodrigues.pdf

SOARES, Lilian Santos; ALMEIDA, Rogéria Comastri de Castro; NUNES, Itaciara Larozza. Conhecimento, atitudes e práticas de manipuladores de alimentos em segurança dos alimentos: uma revisão sistemática. Higiene Alimentar - Vol.30 - no 256/257 - Maio/Junho de 2016.

BRASIL. Ministério da Saúde. Agência Nacional de Vigilância Sanitária. Resolução RDC no 216, de 15 de setembro de 2004. Regulamento técnico de Boas Práticas para Serviços de Alimentação. Diário Oficial da União, Brasília, 16 setembro. 2004. Seção 1, p. 25.

MELO, Aline Gomes de, et al. Conhecimento dos manipuladores de alimentos sobre boas práticas nos restaurantes públicos populares do Estado do Rio de Janeiro. Braz. J. Food Technol., Campinas, v. 13, n. 1, p. 60-68, jan./mar. 2010

SACCOL, Ana Lúcia de Freitas. Sistematização de ferramentas de apoio para boas práticas em serviços de alimentação. 2007. 188 f. Dissertação (Mestrado em Ciência e Tecnologia dos Alimentos) - Universidade Federal de Santa Maria, Rio Grande do Sul, 2007.

SOUZA, Giovanna Carbonera de; SANTOS, Celso Tadeu Barbosa dos; ANDRADE, Anderson Assunção; ALVES, Luciene. Comida de rua: avaliação das condições higiênico-sanitárias de manipuladores de alimentos. Rev. Ciência \& Saúde Coletiva, 20(8):2329-2338, 2015. 
CAVALI, Suzi Barreto; SALAY, Elizabete. Gestão de pessoas em unidades produtoras de refeições comerciais e a segurança alimentar. Rev. Nutr., Campinas, 20(6):657-667, nov./dez., 2007.

ANDREOTTI, Adriana et al. Importância do treinamento para manipuladores de alimentos em relação à higiene pessoal. Iniciação Científica Cesumar - jan-jun. 2003, vol.05, n.01, pp. $29-33$.

SILVA, Jaqueline et al. Enteroparasitoses e onicomicoses em manipuladores de alimentos. Rev Bras Epidemiol 2005; 8(4): 385-92.

MAYRA, Nathiane et al. Condições higiênico-sanitárias das unidades produtoras de alimentos em hotéis do município de Caruaru, Pernambuco. Rev. Veredas Favip, ano 10, vol.7, n. 02, 2014.

MELLO, J. F.; SCHNEIDER, S.; LIMA, M. S.; FRAZZON, J.; COSTA, M. Avaliação de higiene e das boas práticas em UAN. Alim. Nutr.= Braz. J. Food Nutr., v. 24, n.2, p. 175-182, abr./jun. 2013.

ZANDONADI, Renata et al. Atitudes de risco do consumidor em restaurantes de autoserviço. Ver. Nutr., Campinas, 20(1): 19-26, jan/fev. 2007.

OLIVEIRA, Natallya Santos de; GONÇALVES, Thially Braga. Avaliação microbiológica das mãos de manipuladores de alimentos em creches da cidade de Juazeiro do Norte, CE. Revista Interfaces: Saúde, Humanas e Tecnologia, Vol. 3, Nº 1, Ano E, 2015.

CASTRO, Fernanda; BARBOSA, Celso; TABAI, Kátia. Perfil de manipuladores de alimentos e a ótica desses profissionais sobre alimento seguro no Rio de Janeiro (RJ). Revista Brasileira de Economia Doméstica, Viçosa, v. 22, n.1, p.153-170, 2011.

COSTA, Francine Matos; GIARETTA, Andréia Gonçalves; ALVES, Emilaura. Higiene pessoal dos manipuladores de alimentos dos shoppings centers da região da grande Florianópolis. Rev. Técnico Científica (IFSC), v. 3, n. 1 (2012).

Como citar este artigo (Formato ABNT):

FERREIRA, Yuri Gabriel da S.; FOGAÇA, Larissa C. S. Levantamento do nível de conhecimento dos manipuladores de serviços de alimentação em feiras livres sobre Boas Práticas de Fabricação, na Cidade de Vitória da Conquista - BA. Id on Line Revista Multidisciplinar e de Psicologia, 2018, vol.12, n.40, p.1093-1104. ISSN: 1981-1179.

Recebido: 28.05.2018

Aceito: 29.05.2018 